\title{
Lung Cancer Metastasis to the Spine
}

\author{
Ahmet Levent AYDIN 1 , Erhan EMEL ${ }^{2}$, Mehdi SASANI ${ }^{3}$, Cengiz GOMLEKSIZ ${ }^{4}$, Tunc OKTENOGLU³ \\ Ali Fahir OZER ${ }^{5}$ \\ ${ }^{1}$ Istanbul Physical Therapy and Rehabilitation Training Hospital, Neurosurgery Department, Istanbul, Turkey \\ ${ }^{2}$ Bakirkoy Mental and Nervous Diseases Training and Research Hospital, Neurosurgery Department, Istanbul, Turkey \\ ${ }^{3}$ American Hospital, Neurosurgery Department, Istanbul, Turkey \\ ${ }^{4}$ Erzincan University, School of Medicine, Mengücek Gazi Training and Research Hospital, Neurosurgery Department, Erzincan, Turkey \\ ${ }^{5}$ Koc University, School of Medicine, Neurosurgery Department, Istanbul, Turkey
}

\section{ABSTRACT}

In patients affected by malignant tumors, spinal column metastases are frequent. The incidence varies between $30 \%$ and $70 \%$. Lung cancer is the second most frequent cancer invading the spine in women, following breast cancer. All patients are under risk of symptomatic spinal cord compression. The prognosis is poor. Indications for surgery are progressive neurologic deficit, intractable pain, and impending vertebral fracture. Treatment is to preserve neurological functions, promote pain relief and provide functional improvement. Five patients, with symptomatic metastatic spinal cord compression at the thoracic level secondary to lung cancer were operated on. Invasion of the spinal column was from neighbouring lung tissue by direct extension or through segmental arteries. The patients were evaluated regarding neurological status, pain and disability scores, tumor type, level, and surgical procedure. Lung cancer is an aggressive tumor. Patients with symptomatic spinal cord compression must be treated aggressively. With current spinal surgical techniques and a coordinated effort, the life expectancy and quality of these patients are extended.

KEYWORDS: Lung cancer, Vertebral metastases, Thoracic spine

\section{INTRODUCTION}

Cancer is the second leading cause of death and roughly twothirds of cancer patients develop metastases (12). Metastatic disease in the form of distant foci is evident at autopsy in $40 \%$ to $85 \%$ of cases of malignancy. The spine is the most common site of skeletal metastasis. In autopsy series, spinal body metastases are observed in over one third of cancer patients (18). In $5 \%$ of all cancer cases, clinical evidence of spinal cord, cauda equina, or nerve root compromise is found (18). All malignancies may metastasize to the spine. The most common cancers involving the spine are lung, breast, prostate, and renal cell carcinomas (18).

Spinal metastasis was seen in $90 \%$ of prostate, $75 \%$ of breast, $55 \%$ of melanoma, $45 \%$ of lung, and $30 \%$ of renal cell carcinoma patients (18). Symptomatic spinal cord compression is less common and was found in $22 \%$ of breast,
$15 \%$ of lung, and $10 \%$ of prostate cancer patients. The bony lesions in spinal cord metastasis may be either lytic or blastic lesions (18). Lung cancer usually produces lytic bone lesions.

Spinal metastases from a lung cancer may develop at any time during the natural course of lung cancer (18). Metastasis to the spine may be via hematogenous, lymphatic or direct extension. At least $5 \%$ of patients with malignancies suffer from this condition. All these patients suffer from back pain, followed by weakness and ataxia (3).

The clinical aspects of vertebral metastasis depends on the primary tumor. The cause of less frequent neurological deficit in lung metastasis may be severe pain and consequently early surgery for spinal metastasis (16). Metastatic lesions of the spine represent a continuum of the disease process that affects patients with cancer. 
Superior sulcus or pancoast tumors are primary lung tumors. They invade the chest wall and vertebral column and cause specific pain and Horner syndrome $(3,10)$. Classically, metastatic lung tumors invading the spine were mostly considered to be unresectible. The possible treatment modalities are radiotherapy alone or radiotherapy followed by resection. Recent technological advances in spinal instrumentation allow more feasible radical surgery for the treatment of spinal metastasis (3).

Spinal metastasis requires the multidisciplinary efforts of the spine surgeon, radiation oncologist, medical oncologist, orthotist and other health care providers (10).

\section{- CASE REPORTS}

From January 2007 to April 2009, a total of five patients with symptomatic thoracic vertebral invasion secondary to lung cancer underwent palliative surgery in the thoracic and spinal surgery clinics. All of the five patients had direct invasion of the lung cancer to the spine and the indications for surgery were neurological progression due to spinal cord compression and all of them were operated by the same surgical team. All these patients were evaluated regarding the preoperative and postoperative neurological conditions, pain and disability scores, tumor type, tumor level, and surgical procedure. Nine surgical procedures were performed for the five patients. Two patients underwent single operations, two patient underwent two, and the last patient underwent three operations. The reason for the repetitive operations were local recurrences of the tumors. For all the patients, the choice of surgical approach was dependent on the preoperative tumor location. Anterior, posterior or combined anterior-posterior methods of approach such as anterior corpectomy, reconstruction, instrumentation, posterior instrumentation were performed. All the instrumentation procedures were performed after adequate decompression. After the surgery, they were referred to oncology department for local radiotherapy and systemic chemotherapy.

\section{Case 1:}

This 49-year-old male patient was under investigation at the thoracic surgery clinic. His chest radiography demonstrated a superior sulcus tumor at the left side. Examination of a biopsy sample yielded a diagnosis of primary lung carcinoma. Thoracic surgeons were planning pneumectomy while he was referred to neurosurgery clinic because of numbness in both arms, and cervical pain. During the physical examination, the patient experienced severe pain on palpation at the cervico-thoracic junction. In his neurological examination, we discovered hypoesthesia at the T1 and T2 dermatomes. No signs of myelopathy were apparent. Preoperatively, his visual analog score (VAS) was 9 and Oswestry disability index (ODI) was 60. In his thoracic magnetic resonance imaging (MRI) scan, metastatic spinal tumor at the T1 and T2 vertebrae was seen (Figure 1). In December 2007, the patient initially underwent en bloc resection of the upper lobe of the left lung. Than, he was referred to the neurosurgery clinic for the operation of the vertebral metastatic lesions. Under general anesthesia, T1 and T2 corpectomy, stabilization with methylmetacrylate and anterior plating were performed at the first session by

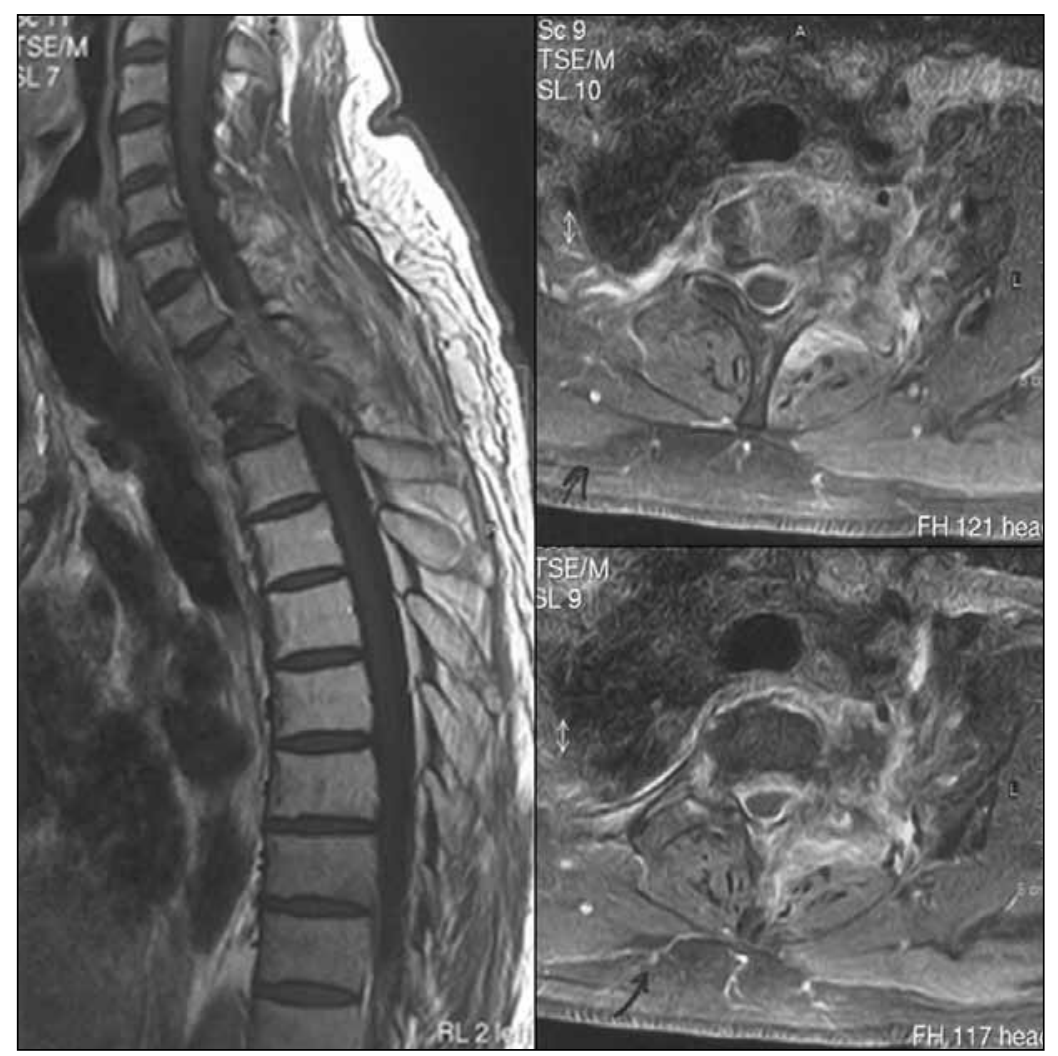

Figure 1: A thoracic MRI scan (sagittal and axial) showed metastatic spinal tumor at the T1 and T2 vertebrae. 


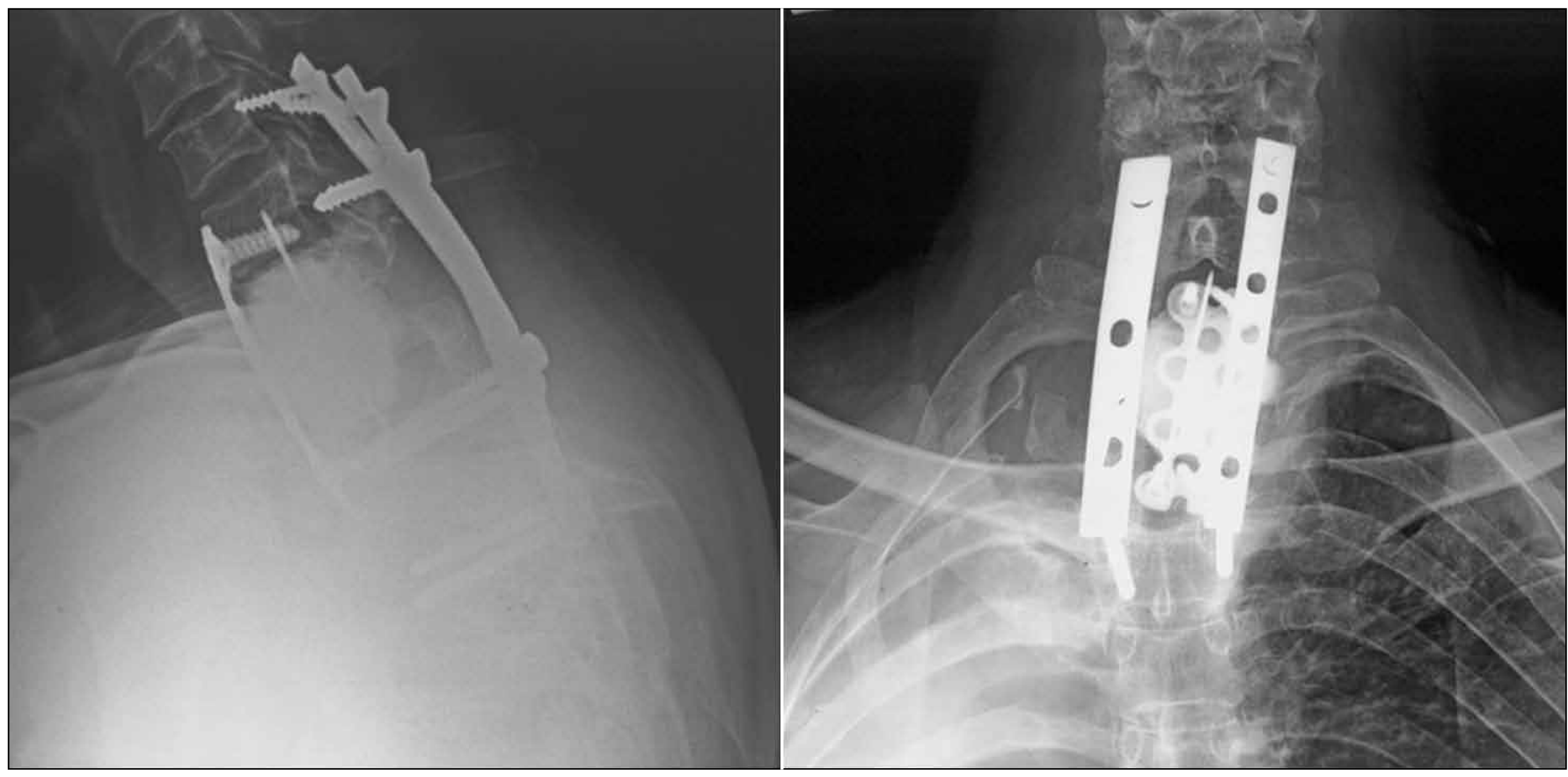

Figure 2: Postoperative plan X-Ray shows total laminectomy, transpedicular screw-rod fixation between C6-7 and T2-3-4 vertebral segments were performed.

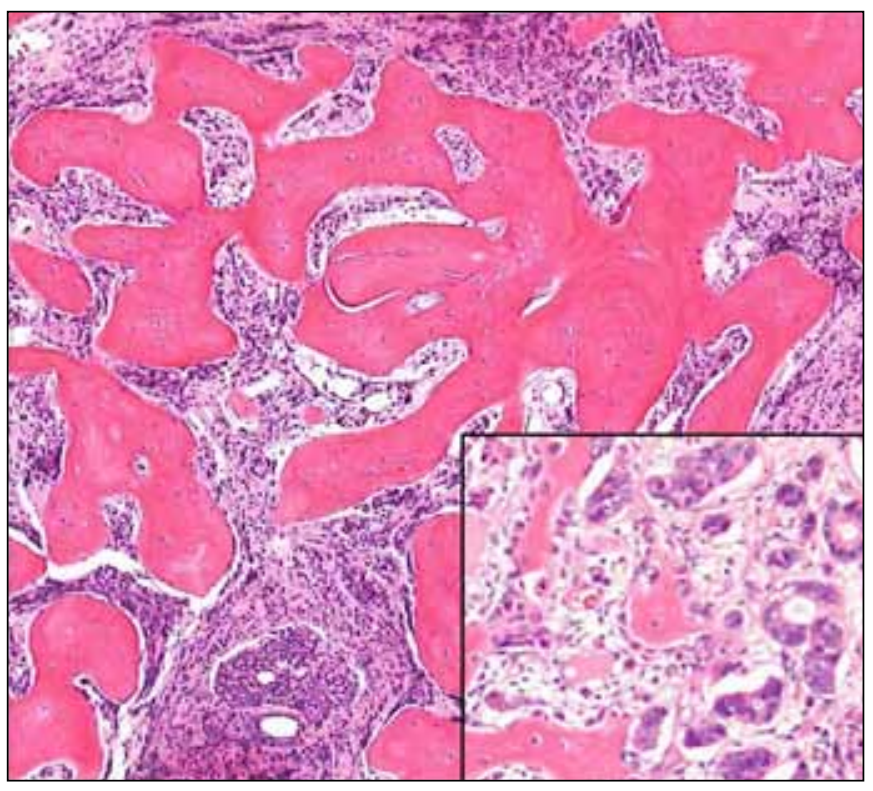

Figure 3: Microscopic view of metastatic lung carcinoma of bone. Carcinomatous glandular structure with osteosclerotic change (Hematoxylin \& Eosin (H\&E), x40 for the main slide; H\&E, x200 at the corner).

an anterior approach. After this procedure, the patient was turned to the prone position with the head fixed with a threepin frame. C6-7-T1-T2-T3-T4 laminas were explored. The $1^{\text {st }}$ and $2^{\text {nd }}$ thoracic laminas were infiltrated by the tumoral tissue. Total laminectomy was applied, and transpedicular screw-rod fixation between C6-7 and T2-3-4 vertebral segments was performed (Figure 2). An allograft was used for fusion. After the hemostasis, the surgery was completed. After the procedure, the patient was followed at the intensive care unit for one day. No neurological deficit was observed after the procedure and he was discharged from the hospital 10 days later and referred to the oncology department. Six months after the operation, his VAS score was 2 and ODI score was 16. Histopathological examination of the tumor specimen came out to be non-small cell carcinoma.

\section{Case 2:}

This case was 38 year-old male patient and was admitted to the hospital in January 2007 with the complaint of dorsal pain. He was under the control of thoracic surgery department with the diagnosis of lung cancer. In 2005, a vertebroplasty operation was performed with the diagnosis of dorsal compression fracture. At the current admission, his physical examination was normal and he had no neurological deficits. His radiological investigations showed metastasis at the $5^{\text {th }}$, $6^{\text {th }}$ and $7^{\text {th }}$ thoracic vertebrae. His preoperative VAS score was 10, ODI was 98. He was operated in February 2007. Posterior partial vertebrectomy to the involved vertebrae and transpedicular screw fixation to the thoracic $3^{\text {rd }}-4^{\text {th }}$, and thoracic $8^{\text {th }}-9^{\text {th }}$ vertebrae was performed. He was discharged 5 days later without complication. In November 2007, he was admitted to the neurosurgery clinic again with the complaint of motor deficit at his hands. In the radiological investigations, intradural extramedullary spinal metastatic tumor was discovered at the seventh cervical and $1^{\text {st }}$ thoracic vertebrae. At the operation, C7-T1 right sided hemilaminectomy and tumor excision was performed. Pathological investigation of the specimen showed metastatic lung carcinoma (adenocarcinoma) (Figure 3) In March 2009, he was admitted 


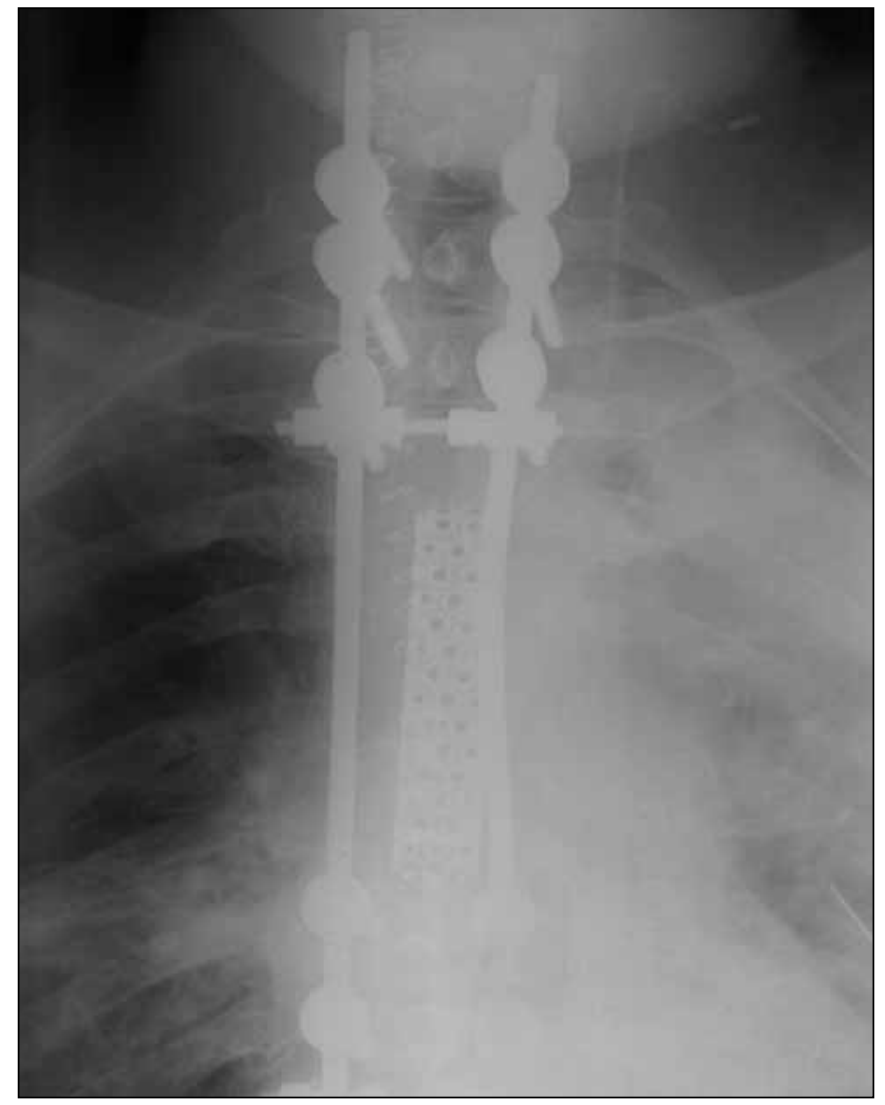

Figure 4: Plain X-Ray (Case 2) shows that thoracotomy, T4-5-6 corpectomy, stabilization with intervertebral cage and afterwards, by the posterior approach, excision of the tumor infiltrating the posterior vertebral elements and posterior transpedicular screw fixation between the $3^{\text {rd }}$ to $10^{\text {th }}$ vertebrae and osteosynthesis were performed. to the hospital with dorsal pain. Metastatic tumor at the T5 and T6 levels was discovered. His neurological examination showed hypoesthesia at the T5 and T6 dermatomes. He was taken to operation. Thoracotomy, followed by T4-56 corpectomy, stabilization with intervertebral cage and afterwards, by posterior approach, excision of the tumor infiltrating the posteror vertebral elements and posterior transpedicular screw fixation between $3^{\text {rd }}$ to $10^{\text {th }}$ vertebrae and osteosynthesis were performed (Figure 4). He was discharged from the hospital 10 days later with no complications. The histopathological investigation of the specimen of this second operation showed metastasis of adenocarcinoma of the lung. Six months after the last operation, his VAS score was 4 , ODI was 24.

\section{Case 3:}

This 84-year-old man was admitted to the hospital with the complaint of difficulty in walking. In his neurological examination, there was paraparesis overt at the right side. In the radiological investigations, there were left sided lung tumor with direct invasion to the vertebral column, and spinal cord compression at the levels of $8^{\text {th }}$ and $9^{\text {th }}$ thoracic vertebrae. He had intractable dorsal pain, with VAS score of 9 and ODI score of 80 . He was operated in January 2008 with the attendance of the thoracic surgeons. Following thoracotomy, superior lobectomy of the left lung and $8^{\text {th }}$ and $9^{\text {th }}$ thoracic vertebrectomy were performed. Histopathological investigation of the specimen showed metastatic non small cell carcinoma (mixed type, adenocarcinoma+squamous cell carcinoma) of lung. Titanium mesh cage and acrylic cement were used for stabilisation (Figure 5). Six months after the operation, most of his pain had disappeared. His postoperative VAS score was 3 , ODI score was 46.

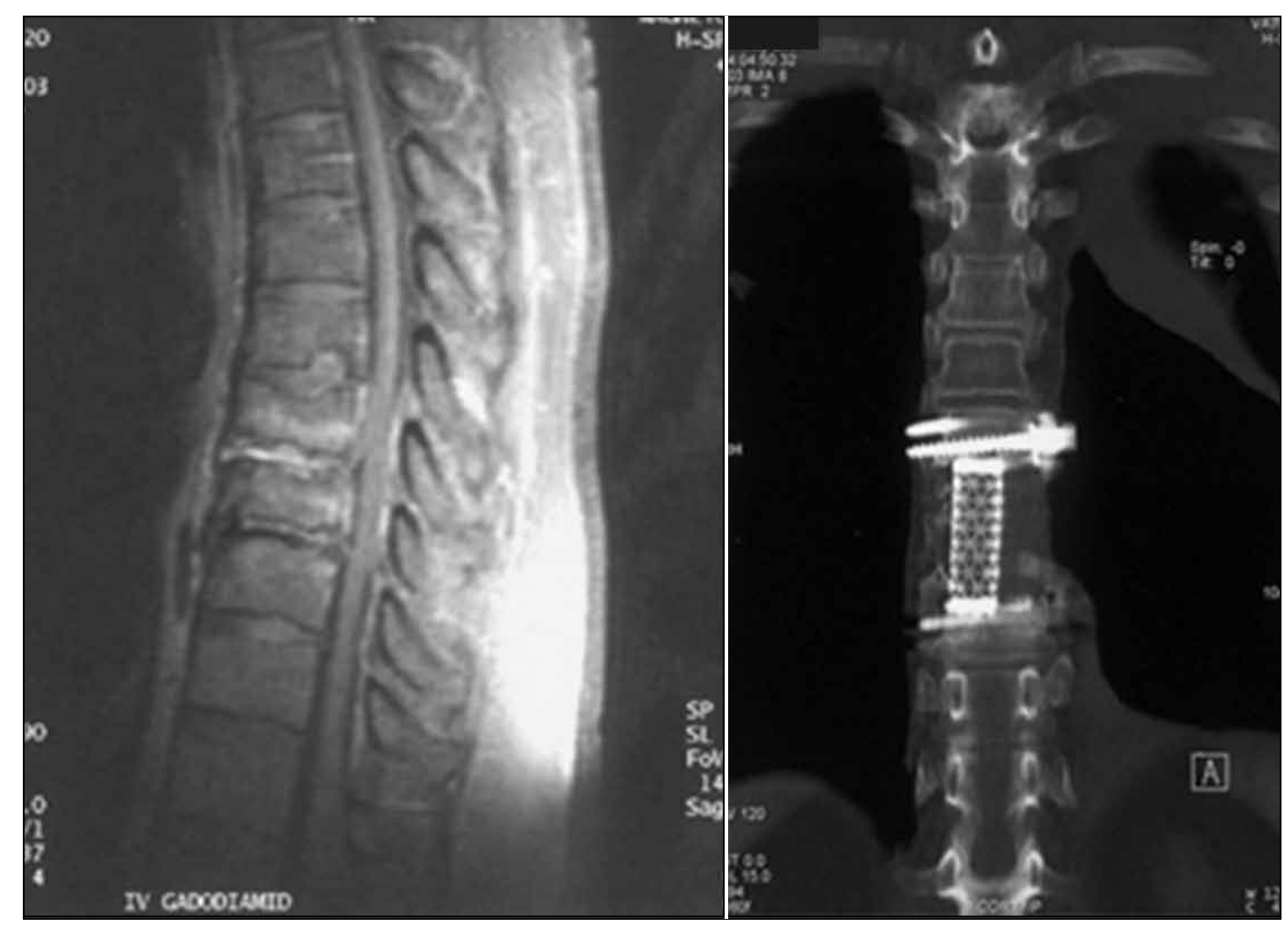

Figure 5: Titanium mesh cage and acrylic cement were used for stabilization. 


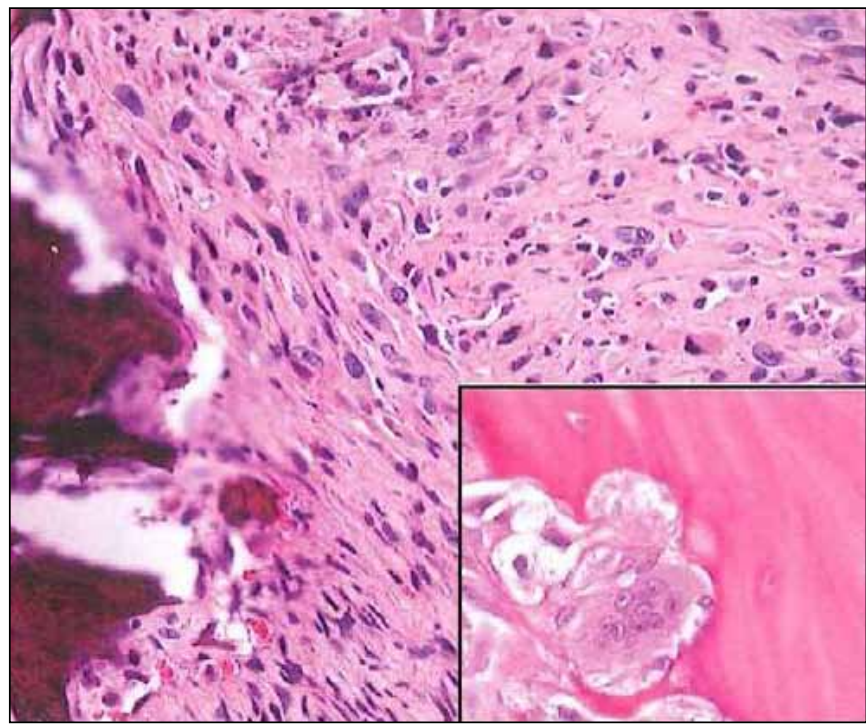

Figure 6: Metastatic non small cell lung carcinoma of bone (sarcomatoid type) (H\&E, x40 for the main slide; H\&E, x200 at the corner).

\section{Case 4:}

This 68-year-old male patient was referred to the thoracic surgery clinic with right lung cancer invading to the dorsal vertebra. He was under chemo- and radiotherapy with the diagnosis of non-small cell (sarcomatoid type) of cancer of lung (Figure 6). After observation of regression in the tumor sizes, he was referred to thoracic and neurosurgery clinics for surgery. In the radiological investigations, right lung superior lobe tumor, with invasion to the vertebral column was shown. He had severe dorsal pain, with VAS score of 9, ODI score of 88. He was operated in March 2009. In the operation, right sided pneumectomy, together with T1, T2 and T3 total laminectomy with posterior approach, total vertebral tumor resection and C4-5-6-7 and T4-5-6-7 posterior instrumentation were performed. He was discharged one week after the operation without neurological deficit. He was nearly pain free 6 months after the operation, with VAS score of 2 , ODI score of 26.

\section{Case 5:}

This 60-year-old male patient had been operated at the thoracic surgery clinic with the diagnosis of lung cancer. The tumor was located at the right lung and right superior lobectomy was performed. The histological diagnosis was squamous cell cancer. Eighteen months after this surgical procedure, he was presented to the same thoracic surgery clinic with the complaint of severe dorsal pain. His VAS score was 10, ODI score was 80 . He had no neurological deficit. His radiological examinations showed recurrence of the tumor at the right bronchial stump, and thoracic vertebral and rib metastasis. After the investigations with MRI, whole body scintigraphy, abdominal computed tomography (CT), positron emission tomography (PET) scan, and histopathological examination of the lymph nodes extirpated by mediastinoscopy, the costal and vertebral metastasis were estimated to be direct invasion. So, re-operation was attempted. In March 2008, he was oper- ated. Following right total pneumectomy and excision of the invaded $5^{\text {th }}$ and $6^{\text {th }}$ ribs, at the same session, $5^{\text {th }}$ and $6^{\text {th }}$ thoracic vertebral corpectomy was performed. Stabilization was made with distractable intercorporal titanium cage and Caneda system (Figure 7A-D). After the operation, his neurological examination was intact. He was discharged from the intensive care unit after 2 days. One week after the operation, he developed postoperative intractable fever of 39-40 degrees centigrade. His microbiological cultural investigations showed proliferation of methicillin-resistant Staphylococcus aureus and pseudomonas microorganisms in his aspirate and hemocultures. In spite of antibiotherapy and supportive treatment, the patient died because of cardiac and pulmonary failures 2 months after the operation. The pathological examination showed squamous cell carcinoma of the lung.

\section{- DISCUSSION}

Surgical treatment and its role in metastatic spinal disease are controversial. Origin of the primary disease is important for the prognosis of the patients (3). Weigel et al. showed that the survival rates in patients with lung cancer were poorest (2.1 months) among the solid cancers (23). However, the life expectancy of lung cancer patients can be prolonged by recent advances in chemotherapeutic agents and targeted therapies (3).

The axial skeleton is the leading site of bone metastases that are caused by hematogenous spread through the rich venous network that drains the lungs, pelvis, and thorax. Breast, lung, prostate and thyroid malignancies account for $50 \%$ to $60 \%$ of metastatic lesions $(14,21,24)$. Breast cancer is the most common source of bony metastasis in women, lung and prostate carcinomas in men. Lung carcinoma commonly metastasizes to the liver, skeleton, bone marrow and brain (16). The extension and size of primary tumor $(\mathrm{T})$, regional lymph node invasion $(\mathrm{N})$ and distant metastasis $(\mathrm{M})$ are taken into consideration in the staging of lung cancers. First described by Denoix in 1946, this TNM staging system has been the major prognostic indicator in these tumors. International Association for the Study of Lung Cancer (IASLC) upgraded the criteria of TNM staging system with the pioneering of International Union Against Cancer (UICC) in 1973. According to the last revisions of TNM staging, lung tumors with chest wall or vertebral metastasis are classified as T3 or T4. Those with vertebral metastasis (T4) are staged as stage 3B. In the non-metastatic group (T1NOM0), 5-year survival rate is about $60 \%$, while it is less than $5 \%$ in T3 or T4 group (13). The vertebral bodies are involved in $80 \%$ of cases of lung cancer metastases. This situation is probably secondary to highly vascular red bone marrow. The thoracic spine is most frequently involved in metastatic disease, followed by the cervical and lumbar regions of the spine. Intramedullary metastases is rarely occur (18). All our cases were accepted to be direct metastasis of the lung tumor to the thoracic spine via neigbourhood.

According to the TNM classification system, all our cases were in T4 group, or stage 3B. According to whole body CT scan, Technetium-99m bone scintigraphy scan and histopathologic 


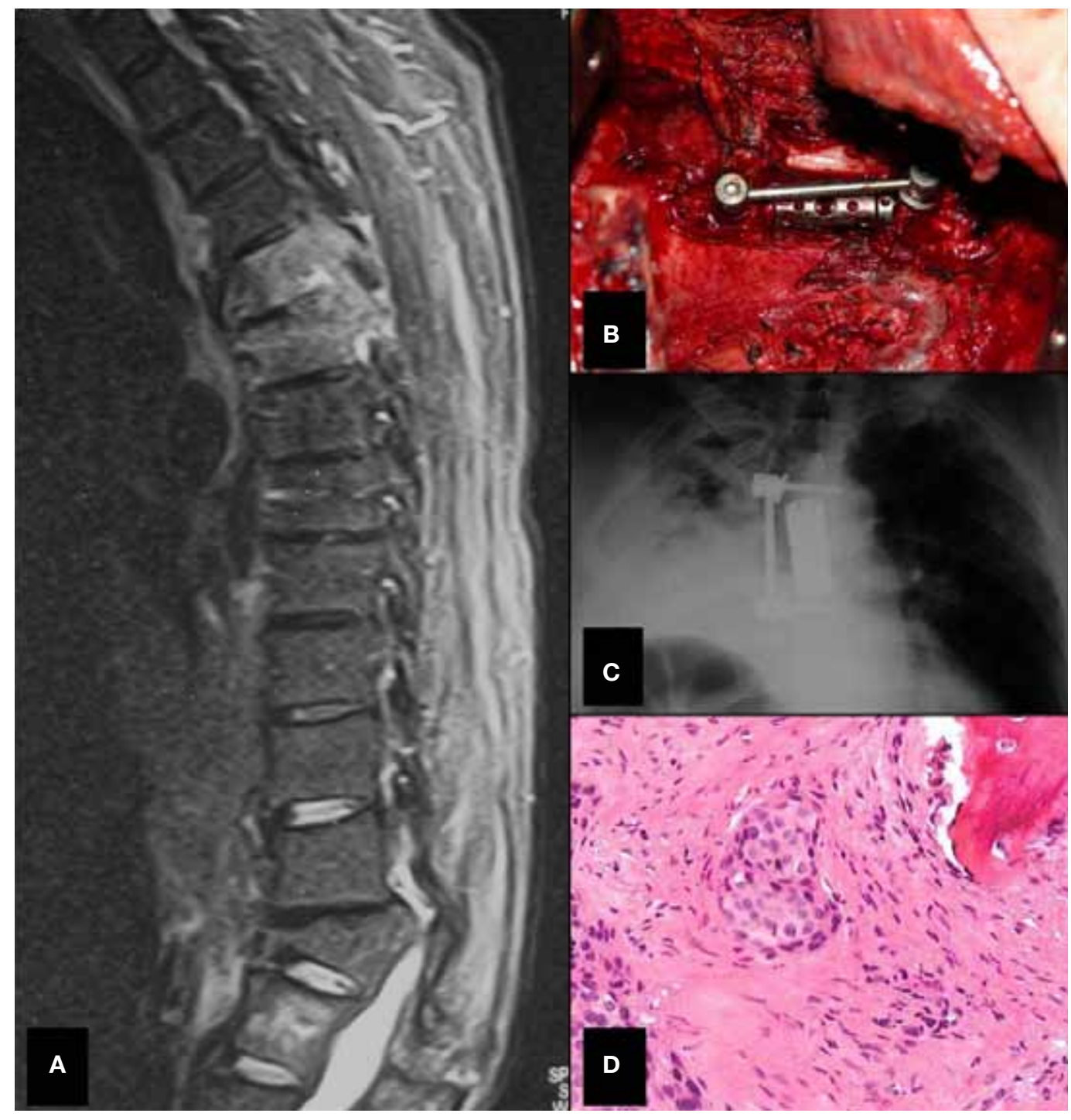

Figure 7: (Case 5)

A) Sagittal MRI,

B) Peroperative Picture,

C) Postoperative plan X-Ray,

D) The histopathological examination shows squamous cell carcinoma of lung. examination of the local and distant lymph nodes, there were no distant lymph node metastases or distant organ metastases. So all the cases were classified as T4N0M0.

Tumors of the lung most commonly occur in the thoracic spine and may seed the thoracic spinal column directly through segmental arteries. Tumors, that involve the spine, metastasize to the spine via either the bloodstream or the lymphatics $(2,6)$. Tumor cells may become implanted in the vertebral column via retrograde flow through Batson's venous plexus. Our cases are considered to metastasize to the vertebral column by direct extension because of close contact of the lung tumor to the vertebral column with metastasis and because of lack of distant organ or lymph node metastasis.

At the time of presentation, back pain occurs in at least $90 \%$ of patients with spinal metastasis of lung cancers (18). As the vertebral body is progressively destroyed, the pain may increase gradually. Back pain may be increased with movement of the spine. Acute onset of pain, either moderate or severe, may indicate a pathological fracture of the vertebral body. This pain may be local, radicular or axial. Local pain is secondary to mass effect of the tumor or stretching and distortion of the periosteum from the tumor destruction (18). Local pain is constant, and is not increased by movement. Radicular pain is secondary to nerve root compression by epidural tumor extension. Axial pain is mechanical in nature and arises from a structural abnormality of the spinal column, and may indicate instability. It may worsen with movement. (18).

Neurological deficits such as paraparesis or mild weakness of the extremities, may be seen in patients with spinal metastasis. In pathological spinal fractures or fracture/dislocations, an acute neurological deterioration may occur (18). Therefore, patients with systemic malignancy and neurological deficits requires urgent radiological evaluation for spinal metastasis (18). In our series, patients were mainly presented with dorsal pain. Only the third patient was presented with paraparesia. There was a great recovery of the patients based on the comparison of pain scores. The first four patients were almost 
pain free for 6 months after the operations. The fifth patient died 2 months after the operation because of cardiac and pulmonary failure on the basis of systemic infection. Patient evaluation will usually incorporate $\mathrm{x}$-rays, MRI, and CT scanning. X-rays are usually used to detect the metastatic involvement of the spine when suspected. Spinal alignment and stability may be assessed by dynamic X-rays. However, these films are not enough to rule out vertebral body metastases. Vertebral body destruction is more prominent and easily visualized in cervical spine involvement. Pedicle erosion is one of the common radiological findings in the thoracic and lumbar spine metastasis (18). Compression fractures may also be seen in spinal metastasis. These lesions often spare the disc spaces and it is easy to differentiate from the pyogenic spinal infections (18). Technetium-99m bone scintigraphy is more sensitive than plain films in detecting spinal bone metastases.

CT is sensitive for bone destruction in vertebral column. It is easy to identify the extent of bony involvement. Multiple sites of bony involvement may be also detected by whole spine CT scan. MRI is another the imaging method for the evaluation of spinal metastases. Neural and spinal cord compression may be detected by MRI scan and the whole spine can be scanned with multiple planes by MRI and paravertebral tumor extension and delineation of tumor extent may be assessed by the same method. MRI is also sensitive for bone marrow involvement by a systemic malignancy. Most metastatic lesions appear hypointense on T1-weighted images and hyperintense on T2weighted images of the spinal MRI. Gadolinium enhancement may obscure some metastatic bone lesions because of the normal vertebral body enhancement. Informations obtained from the MRI and CT scans are important for surgical planning (18).

The histological types of lung cancers are squamous, adenocarcinoma, and small cell carcinoma. Non-small cell carcinoma has the best overall prognosis and longer survival rate, thus patients have the highest likelihood of the development of skeletal metastases.

The treatment of lung carcinoma is dependent on the type of tumor. Small cell lung carcinoma is more responsive to chemotherapy and radiation therapy (6). Histological diagnosis of our patients were as follow; metastatic non-small cell carcinoma (mixed type) of the lung in cases 1 and 3 , adenocarcinoma in case 2 , non-small cell sarcomatoid type in case 4 , and squamous cell carcinoma in case 5 . Patients with metastatic spinal tumors were considered to be at the terminal stage of the disease. Only palliative treatments were performed.

Two-year survival rate with radiation therapy was no more than $15 \%$ in patients with lung cancer located in the superior sulcus and spine invasion $(8,9)$. Survival period of patients with metastatic tumors is longer today and more durability of surgery has been reported thanks to the advances of chemo-, immuno-, and radiotherapy in malignant tumors (9).

Radical vertebral surgery may be considered in patients with spinal metastases who had acute progressive neurological deficits, intractable pain, and fracture of the vertebral body compressing to the spinal cord. The goal of surgical treatment is to relieve pain and prevent or reverse neurological functions as soon as possible. Meanwhile, each patient with spinal metastasis is different, and the indications and contraindications should be individualized. Patients with solitary spinal metastasis, who had a controllable primary tumor without invasion to the adjacent visceral organs or distant metastases, are candidates for radical spinal surgery (19) All the 5 patients in our series were in this group.

Indications for surgery are presence of primary focus where CT-guided biopsy is not possible, progressive neurological deficits despite conservative treatment such as radiotherapy and chemotherapy, instability due to bony destruction, and spinal cord compression by the bone fragments. Axial pain is also an indication for stabilization surgery in spinal metastasis (18). It is difficult to regain neurological functions after decompressive surgery in patients with complete absence of motor and sensory functions longer than 12 hours (18).

The expected complications of this surgery are bronchopleural fistulas, pneumonia, cerebrospinal fistulas, postoperative infections, implant failure due to the osteoporotic effect of chemo- and radiotherapy, and neurological injury. Among the metastatic lung cancers of the spine, those with direct invasion deserve special care in the era of spinal surgery. Considering this group of spinal metastasis, a complete resection, followed by reconstruction and a multidisciplinary approach must be carried out to achieve long-term survival $(1,7,9,11$, 20).

Since the report of Okada et al. in 1985 (15), Komagata presented seven local lung cancer metastasis cases to the vertebra with partial or complete resections, using anterior, posterior or lateral approaches $(10,15)$. Complete removal of vertebrae and surrounding tissue and multilevel laminectomy is recommended in local invasion of the vertebra. With the recent advances in spinal surgery, corpectomy or vertebrectomy is more feasable. The decision to use the particular surgical approach is dependent on the location of the tumor. If tumor invasion extends to the spinal canal, transverse process, facet joints, or lamina must also be removed (8). According to Weigel et al, the anterior spinal approach is better for decompression (23). Anterior approaches, together with resection of the PLL and unilateral pedicle on the side of thoracotomy provide exposure to decompression. For circumferential epidural tumors, combined anteroposterior approach is usually necessary (3).

DeMeester et al. reported successful surgical treatment of patients with spinal in whom en bloc vertebrectomy and removal of adjacent tissue is required $(4,9)$. Sundaresan et al. recommended anteroposterior resection of spinal metastasis in order to achieve total tumor resection (22). Prasad and Schiff are also recommended anteroposterior resection combined with instrumentation for an effective surgical treatment (17).

The complication rate may be high in patients who undergo combined anteroposterior surgery $(3,17,22)$. According to Koizumi et al., total vertebrectomy is required in patients who underwent hemivertebrectomy for apical chest tumors 
in case $50 \%$ or more of the vertebra is destroyed (9). Some surgeons perform hemi- and total vertebrectomy for a complete resection. They recommend complete resection to control local recurrence of spinal invasion (9). A vertebral body with less than $30 \%$ involvement may be treated with partial vertebrectomy, but it is difficult to determine clear surgical margins with partial tumor resection (9).

The local recurrence rate is still high despite the surgical treatment modalities. Therefore, additional adjuvant chemoradiotherapy must be performed to control tumor recurrence. Preoperative radiation therapy, in combination with postoperative concurrent chemoradiotherapy is effective to control microscopic residual tumors (9). It is stated that even microscopic residual tumor tissue worsens the prognosis so much that the prognosis of incompletely resected tumors and non-operated tumors are similar. (5). In all our cases, total vertebrectomy was performed. The histopathological examination of the vertebrectomy material showed tumor-free borders in all cases. In conjunction with instrumentation, postoperative radio- and chemotherapy were applied in oncology department.

\section{- CONCLUSION}

In lung metastasis to the spine, adequate decompression and stabilization are the cornerstones of good surgical outcome. The choice of approach mostly depends on the tumor topography, histopathological nature of the tumor and the neurological condition of the patient. With the recent developments in spinal and thoracic surgery, complete resection of the involved vertebral segments, together with postoperative radiotherapy and chemotherapy will provide longer and more comfortable life and pain-free survival time.

\section{REFERENCES}

1. Anderson TM, Moy PM, Holmes EC: Factors affecting survival in superior sulcus tumors. J Clin Oncol 4:1598-1603,1986

2. Batson OV: The function of vertebral veins and their role in the spread of metastases. Ann Surg 112:138-139, 1940

3. Chen YJ, Chang GC, Chen HT, Yang TY, Kuo BI, Hsu HC, Yang HW, Lee TS: Surgical results of metastatic spinal cord compression secondary to non small lung cancer. Spine 32: 413-418, 2007

4. DeMeester TR, Albertucci M, Dawson PJ, Montner SM: Management of tumor adherent to the vertebral column. $J$ Thorac Cardiovasc Surg 97: 373-378, 1989

5. Downey RJ, Martini N, Rusch VW, Bains MS, Korst RJ, Ginsberg RJ: Extent of chest wall invasion and survival in patients with lung cancer. Ann Thorac Surg 68:188-193, 1999

6. Ghanayem AJ: Metastatic tumors of the spine. In: Vaccaro AR, Betz RR, Zeidman SM (eds). Principles and Practice of Spine Surgery. Philadelphia, PA: Mosby, 2003:213-222

7. Ginsberg RJ, Martini N, Zaman M, Armstrong JG, Bains MS, Burt ME, McCormack PM, Rusch VW, Harrison LB: Influence of surgical resection and brachytherapy in the management of superior sulcus tumor. Ann Thorac Surg 57:1440-1445, 1994
8. Gokaslan ZL, York JE, Walsh GL, McCutcheon IE, Lang FF, Putnam JB Jr, Wildrick DM, Swisher SG, Abi-Said D, Sawaya $\mathrm{R}$ : Transthoracic vertebrectomy for metastatic spinal tumors. J Neurosurg 89:599-609, 1998

9. Koizumi K, Haraguchi S, Hirata T, Hirai K, Mikami I, Yamagishi S, Okada D, Kinoshita H, Enomoto Y, Nakajima Y, Shimizu K: Surgical treatment of lung cancer with vertebral invasion. Ann Thorac Cardiovasc Surg 10: 229-234, 2004

10. Komagata $M$, Nishiyama $M$, Imakiire $A$, Kato $H$ : Total spondylectomy for en bloc resection of lung cancer invading the chest wall and thoracic spine. J Neurosurg Spine 100: 353-357, 2004

11. Maggi G, Casadio C, Pischedda F, Giobbe R, Cianci R, Ruffini E, Molinatti M, Mancuso M: Combined radiosurgical treatment of Pancoast tumor. Ann Thorac Surg 57:198-202, 1994

12. Masaryk TJ: Neoplastic disease of the spine. Radiol Clin North Am 29:829-843, 1991

13. Mountain CF: Revisions in the international system for staging lung cancer. Chest (American College of Chest Physicians) 111: 1710-1717, 1997

14. O'Connor MI, Currier BL: Metastatic disease of the spine. Orthopedics 15: 611-620, 1992

15. Okada K, Kato H, Yamanaka A. Resection of the vertebrae in a patient with lung cancer invading the spine. Geka Rinshou 1:61-66, 1985

16. Onimus M, Papin P, Gangloff S: Results of surgical treatment of spinal thoracic and lumbar metastases. Eur Spine J 5:407411, 1996

17. Prasad D, Schiff D: Malignant spinal cord compression. Lancet Oncol 6:15-24, 2005

18. Ratliff JK, Cooper PR: Metastatic spine tumors. Southern Med J 97: 246-253, 2004

19. Sakaura H, Hosona N, Mukai Y, Ishii T, Yonenobu K, Yoshikawa $\mathrm{H}$ : Outcome of total en bloc spondylectomy for solitary metastasis of the thoracolumbar spine. J Spinal Disord Tech 17: 297- 300, 2004

20. Sartori F, Rea F, Calabro F, Mazzucco C, Bortolotti L, Tomio $\mathrm{L}$ : Carcinoma of the superior pulmonary sulcus. Results of irradiation and radical approach. J Thorac Cardiovasc Surg 104:679-683, 1992

21. Silverberg E, Lubera JA: Cancer statistics. Cancer 38:5-22, 1988

22. Sundaresan N, Sachdev VP, Holland JF, Moore F, Sung M, Paciucci PA, Wu LT, Kelligher K, Hough L: Surgical treatment of spinal cord compression from epidural metastasis. J Clin Oncol 13: 2330-2335, 1995

23. Weigel B, Maghsudi M, Neumann C, Kretschmer R, Müller FJ, Nerlich M: Surgical management of symptomatic spinal metastases: Postoperative outcome and quality of life: Spine 24: 2240-2260, 1999

24. Wolcott WP, Malik JM, Shaffrey Cl: Differential diagnosis of surgical disorders of the spine. In: Benzel EC (ed). Spine Surgery Techniques, Complications, Avoidance and Management. Philadelphia: Elsevier Churchill Livingstone, 2005:33-60 